\title{
BMJ Open Patient Reporting of Safety experiences in Organisational Care Transfers (PRoSOCT): a feasibility study of a patient reporting tool as a proactive approach to identifying latent conditions within healthcare systems
}

\author{
Jason Scott, ${ }^{1}$ Justin Waring, ${ }^{2}$ Emily Heavey, ${ }^{1}$ Pamela Dawson ${ }^{1}$
}

\begin{abstract}
To cite: Scott J, Waring J, Heavey E, et al. Patient Reporting of Safety experiences in Organisational Care Transfers (PRoSOCT): a feasibility study of a patient reporting tool as a proactive approach to identifying latent conditions within healthcare systems. BMJ Open 2014;4: e005416. doi:10.1136/ bmjopen-2014-005416
\end{abstract}

- Prepublication history for this paper is available online. To view these files please visit the journal online (http://dx.doi.org/10.1136/ bmjopen-2014-005416).

Received 7 April 2014 Accepted 17 April 2014

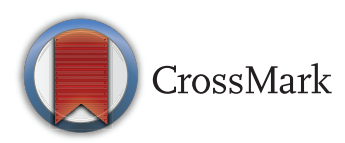

${ }^{1}$ Faculty of Health \& Life Sciences, York St John University, York, UK ${ }^{2}$ Centre for Health Innovation, Leadership and Learning, Nottingham University Business School, Nottingham, Nottinghamshire, UK

Correspondence to Dr Jason Scott; j.scott@yorksj.ac.uk

\section{ABSTRACT}

Background: It is increasingly recognised that patients can play a role in reporting safety incidents. Studies have tended to focus on patients within hospital settings, and on the reporting of patient safety incidents as defined within a medical model of safety. This study aims to determine the feasibility of collecting and using patient experiences of safety as a proactive approach to identifying latent conditions of safety as patients undergo organisational care transfers.

Methods and analysis: The study comprises three components: (1) patients' experiences of safety relating to a care transfer, (2) patients' receptiveness to reporting experiences of safety, (3) quality improvement using patient experiences of safety. (1) A safety survey and evaluation form will be distributed to patients discharged from 15 wards across four clinical areas (cardiac, care of older people, orthopaedics and stroke) over 1 year. Healthcare professionals involved in the care transfer will be provided with a regular summary of patient feedback. (2) Patients $(n=36)$ who return an evaluation form will be sampled representatively based on the four clinical areas and interviewed about their experiences of healthcare and safety and completing the survey. (3) Healthcare professionals $(n=75)$ will be invited to participate in semistructured interviews and focus groups to discuss their experiences with and perceptions of receiving and using patient feedback. Data analysis will explore the relationship between patient experiences of safety and other indicators and measures of quality and safety. Interview and focus group data will be thematically analysed and triangulated with all other data sources using a convergence coding matrix.

Ethics and dissemination: The study has been granted National Health Service (NHS) Research Ethics Committee approval. Patient experiences of safety will be disseminated to healthcare teams for the purpose of organisational development and quality improvement. Results will be disseminated to study participants as well as through peer-reviewed outputs.

\section{Strengths and limitations of this study}

- A strength is that the study will demonstrate how, or if, routinely collected data on patient experiences of safety during an organisational care transfer is linked to other measures and indicators of quality and safety.

- A unique feature of the investigation will be how patient feedback on safety promotes organisational learning within multiple healthcare providers when patients' care crosses healthcare boundaries.

- Challenges include engaging patients to report on their experiences of safety, and engaging healthcare professionals to distribute surveys and use feedback for quality improvement.

\section{BACKGROUND}

Patient safety has received much attention since the publications of An Organisation with a Memory $^{1}$ in the UK and To Err is Human ${ }^{2}$ in the USA at the turn of the century. Since then, there has been a particular emphasis on identifying patient safety incidents, broadly defined as an incident that harmed or had the potential to harm a patient. ${ }^{3}$ The most prominent approach to identifying unsafe care is the use of incident reporting, where healthcare professionals are required to identify and report on safety incidents. ${ }^{4}$ However, there have been a number of criticisms of healthcare professional incident reporting, with healthcare professionals not engaging with incident reporting due to a myriad of cultural issues ${ }^{5}$ and subsequently under-reporting incidents. ${ }^{6} 7$ This is of particular importance to care transfers between organisations, which are acknowledged to be especially high in risk ${ }^{8-11}$ and are often haphazard. ${ }^{12}$ 
When willing and able, ${ }^{13}$ patients are believed to have a role in improving their own safety during care transfers, which includes the identification and reporting of their own safety ${ }^{14}$ and increased involvement in the handover process itself. ${ }^{15}$ Recent reports in the UK, such as the Francis Inquiry ${ }^{16}$ and the report by Donald Berwick, ${ }^{17}$ indicate that patients should be involved at all levels in their own safety. This involvement falls into three categories: informing a management plan, monitoring and ensuring safe delivery of treatment and making systems safer, ${ }^{18}$ the latter of which includes reporting on experiences of safety.

Patients differ from healthcare professionals in their perceptions of safety, ${ }^{19-21}$ and these differences need to be addressed. By involving patients in their own safety, healthcare professionals can encourage them to act as an extra safeguard within the healthcare system, ${ }^{14}$ in line with the systems approach to safety. ${ }^{4}$ Where the definitions of safety differ between the patient and healthcare professional, it is only the patient who can identify and report on feeling unsafe in relation to their own definition of safety.

As such, the aim of this study is to determine the feasibility of implementing a patient safety survey which will measure patients' experiences of their own safety relating to care transfer, in particular the discharge, journey and arrival stages of the transfer. In order to achieve this aim, specific objectives include:

1. Measurement of experiences of safety relating to a care transfer following discharge from hospital, including a comparison of how these experiences relate to current measures and indicators of safety (readmission rates, length of stay, Datix reports on patient experience and safety thermometer data).

2. Determining response rates and investigating the receptiveness of patients to the reporting tool, including the applicability of the domains of safety measured.

3. Investigating the engagement and responsiveness of healthcare teams to the reporting tools and reports of safety, and the promotion of organisational learning.

\section{METHODOLOGY}

Appreciative Inquiry (AI) is a methodological approach to research and organisational development that focuses on 'what works well' in an organisation, as opposed to what does not work. ${ }^{22-24}$ It has been acknowledged that trends in adverse events do not appear to be improving, ${ }^{25}$ and there is an increasing emphasis, particularly in proactive approaches to safety management, that effective safety management requires identifying and measuring 'safety', as opposed to 'unsafety'. ${ }^{26}$ Utilising principles of AI provides a methodological basis for the focus on 'safe', as opposed to 'unsafe' care. The involvement of patients in this proactive approach with an emphasis on safe care provides a radical alternative to existing methods of measuring safety. By taking this positive approach, AI has the ability to engage healthcare professionals by shifting the focus from problems to solutions. ${ }^{27}$

\section{METHODS}

The study will investigate patient reports of safety following a care transfer, which will include the discharge process, the journey and the arrival or admission process at the following organisation. The safety surveys will be distributed to patients discharged from wards in four clinical areas: cardiac, care of older people, orthopaedics and stroke. These clinical areas were chosen in consultation with patient safety teams at the research sites so as to focus on clinical areas predominantly associated with older patients who are at increased risk of safety incidents $^{28}$ and are recognised as high priorities. ${ }^{29}$ Furthermore, the safety survey was originally developed based on older patients' perceptions of safety in care transfers. ${ }^{14}$

\section{Study design}

The study will utilise a mixed methods approach, with qualitative and quantitative data collected across three components of the study. The three components reflect the objectives of the study, and include (1) patients' experiences of safety relating to a care transfer, (2) patients' receptiveness to reporting experiences of safety and (3) quality improvement using patient experiences of safety (figure 1 ).

Qualitative methods will supplement quantitative data collected from the safety surveys, which will provide an understanding of the processes involved in learning from the patient feedback, potentially leading to quality improvement. The survey results will be made available to clinicians, thus allowing them to take a proactive approach to safety. This will consist of implementing a bi-weekly feedback loop between the research team and healthcare professionals involved in the transfer of patients. The feedback will be provided bi-weekly in the form of a clinical dashboard collated from the safety surveys representing three categories: patients within their own healthcare unit, patients within their clinical area and patients across the study (unidentifiable outside of their healthcare unit; figure 2).

\section{Safety survey for organisational care transfers}

The safety survey was developed by healthcare professionals and expert patients. It provides a brief explanation of patient safety and captures patient reports of safety across three stages of the care transfer (discharge, journey and arrival or admission). The questions asked in each stage of the care transfer are provided in table 1 , and focus around six domains: communication, responsiveness, waiting times, falls, medication and hygiene. These domains are based on patient perceptions of safety. ${ }^{14}$ Patients or their carers are asked to report on three 
Distribution of safety survey

Safety Survey distributed to patients ( $n=$ approx. 14,000) at point of discharge from wards $(n=15)$

- Readmission rates, length of stay, Datix ${ }^{\text {Tw }}$ reports of safety, safety thermometer data

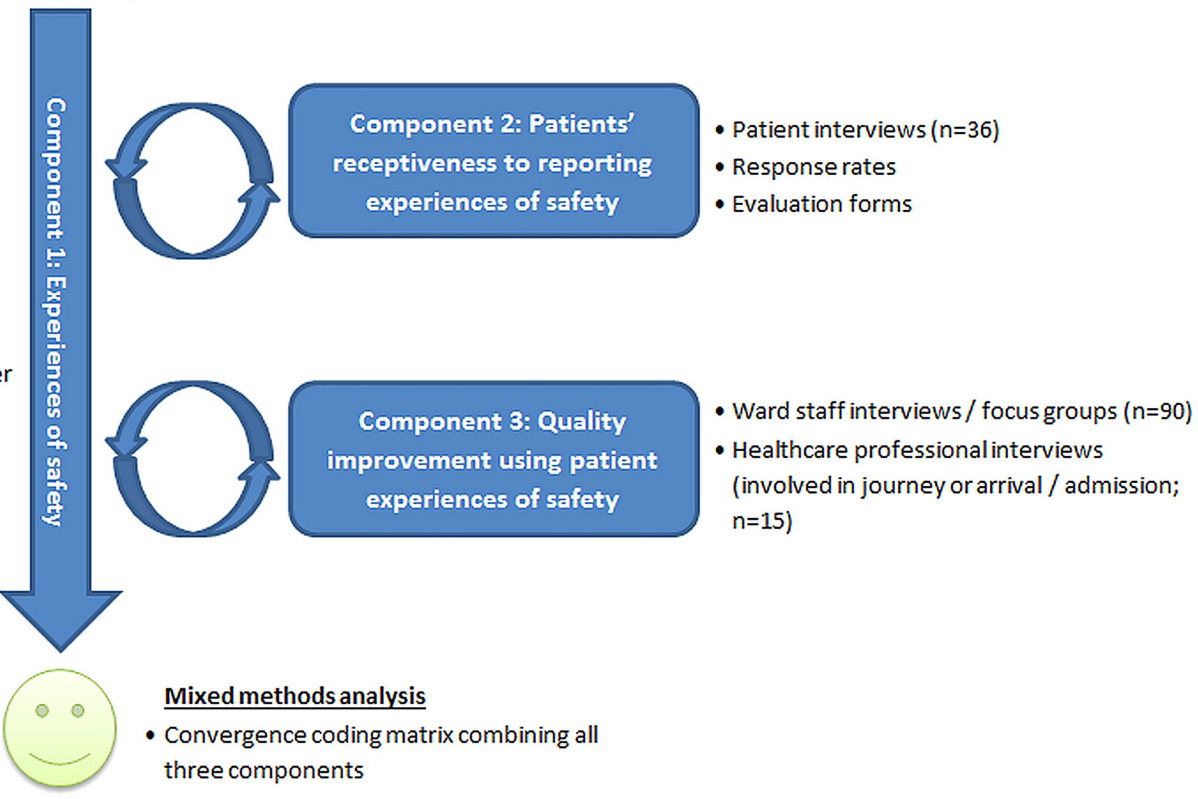

Figure 1 Flowchart demonstrating how the components of the study interlink.

levels of safety: safe (green), neutral (yellow) and unsafe (red), and to leave any non-applicable sections blank.

The safety survey asks patients or carers the mode of transport for the journey and the arrival destination. Respondents are also asked if there was another reason they felt safe or unsafe and if anything could have been done to make the patient feel safer; the safety survey gives space to explain their answers to these questions. The survey also provides patients or carers with the contact details of the lead researcher, so that patients can ask questions about the survey.

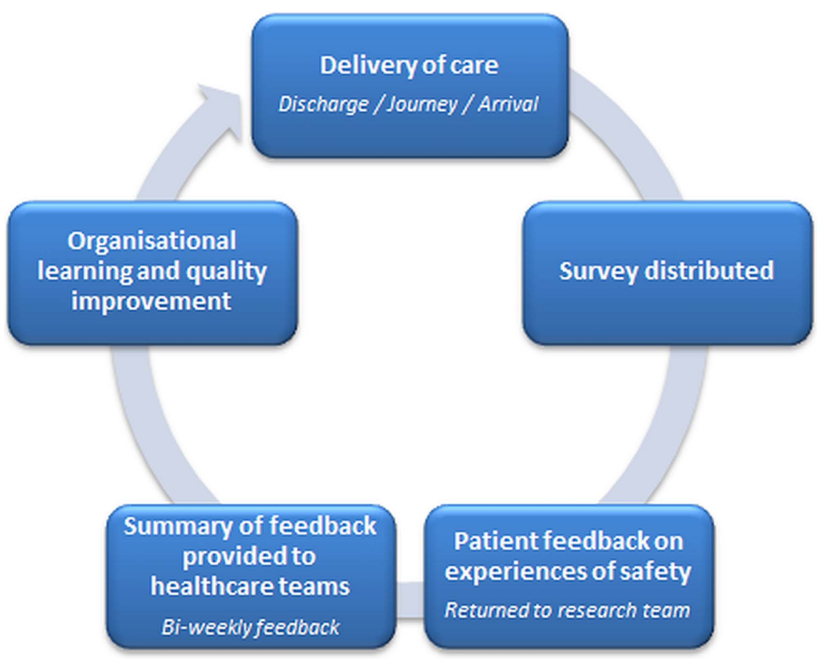

Figure 2 Process of feedback to healthcare teams, organisational learning and quality improvement.

\section{Study setting}

Surveys will be distributed by ward staff in three hospitals across two National Health Service (NHS) trusts. The four clinical areas will be represented across 15 wards: cardiac $(n=3)$, care of older people $(n=4)$, orthopaedics $(n=4)$ and stroke $(n=4)$. Distribution of numbered surveys by ward staff means that it is known where the patient has been discharged from. During the course of the study, the safety surveys will be used to map how patients are transported (journey) and who their care is handed over to (arrival or admission).

\section{Component 1: patients' experiences of safety relating to an organisational care transfer \\ Objective}

Measurement of experiences of safety relating to a care transfer following discharge from hospital, including a comparison of how these experiences relate to current measures and indicators of quality and safety.

\section{Method}

Patients will be provided with a safety survey on discharge from hospital. Surveys are a commonly used method of capturing feedback from patients on a wide number of healthcare topics. Of relevance to this study are patient experience surveys and patient safety surveys, with the latter currently the most reported method of capturing patient reports of safety. ${ }^{13}{ }^{30}$ Routinely collected data relating to measures and indicators of quality and safety will also be collected. 
Table 1 Questions asked in the safety survey about the different stages of the care transfer

\begin{tabular}{|c|c|c|}
\hline \multicolumn{3}{|c|}{ Please tick which of the following affected how safe or unsafe you felt } \\
\hline Discharge & Journey & Arrival or admission \\
\hline Communication from staff & Communication from staff & Communication from staff \\
\hline Staff listening to you & Staff listening to you & Staff listening to you \\
\hline Departure running to schedule & Journey running to schedule & Waiting times \\
\hline Falling or potential falls & Falling or potential falls & Falling or potential falls \\
\hline Medication problems or concerns & Medication problems or concerns & Medication problems or concerns \\
\hline Hygiene & Hygiene & Hygiene \\
\hline \multicolumn{3}{|c|}{$\begin{array}{l}\text { Please use this space to tell us if there was another reason why you felt safe or unsafe or to expand on your answers above. } \\
\text { What could we have done to make you feel safer during your transfer? }\end{array}$} \\
\hline
\end{tabular}

\section{Sample}

All patients discharged from the included wards, or their carers, will be given a safety survey by ward staff. Owing to the nature of the wards, it is not anticipated that this sample will include any patients under the age of 18. As all patients will be given the safety survey, no power calculation was conducted; instead response rates will be monitored to inform feasibility for the universal invitation for patients to provide feedback on their safety in the setting of care transfers. It is estimated, based on discharge information from the sites, that the safety survey will be distributed to 14000 patients over a 12-month period. Routinely collected data will consist of readmission rates, length of stay, Datix reports on patient experience and safety thermometer data for each of the wards included in the study.

\section{Procedure}

The safety survey will be given to patients by an appropriate member of the clinical team or an administrator responsible for compiling discharge information, for example, discharge coordinator or ward clerk. Patients will be given a letter of invitation to the study, the safety survey and an evaluation form. The evaluation form will invite patients to evaluate aspects of the safety survey, including its relevance to their own experiences of safety, its ease of use and its helpfulness in educating them about patient safety. The forms will be used to guide the ongoing development of the safety survey. Patients will also be given a prepaid addressed envelope, a technique which has been shown to improve response rates to surveys. ${ }^{31}$ Those distributing the safety survey will be asked to prompt the patient to complete and return the safety survey.

Participants will opt-in on completion and return of the safety survey and/or an evaluation form. The option to return either is designed to reduce bias from those who evaluate the safety survey negatively or do not wish to complete it. Patients' family members or carers will be encouraged to assist the patients to complete the survey where applicable, or to complete it on their behalf.

Each return envelope will have a unique identifying number which will be used to identify the ward that the patient was discharged from. Owing to the potentially sensitive nature of the topic, patients will have the option of returning the safety survey anonymously, and the identifying number will not be able to be used to identify individual patients.

\section{Analysis}

Descriptive and inferential statistical analysis will determine if a relationship exists between patient experiences of safety and the other indicators and measures of quality and safety (Datix reports, safety thermometer data, readmissions, length of stay). One-way between-group analysis of variance will be used to compare response rates between sites. Non-parametric tests (in particular Kruskal-Wallis test and Spearman's r) will be used to analyse differences and relationships in evaluation responses. Descriptive statistics will form a mapping process of the journey and admissions stages of the transfer, which will be used in component 3 . Evaluations of the safety survey will be used to modify the reporting tool on a monthly basis to ensure it meets the needs of the patients.

\section{Component 2: patients' receptiveness to reporting experiences of safety \\ Objective}

To determine response rates and investigate the receptiveness of patients to the reporting tool, including the applicability of the domains of safety measured.

\section{Method}

Semistructured interviews, incorporating appreciative and cognitive interview components, will be conducted with patients to investigate their experiences of safety in care transfers and their experiences of providing feedback on their safety. Appreciative interviews will utilise the discover and dream stages of $\mathrm{AI}^{32}$ to investigate experiences of, and ideas about, safety. Cognitive interviews will involve inviting interviewees to describe their thought processes in response to survey questionnaires, in order to identify any potential misunderstandings or other problems with those questionnaires; such interviews have proven useful in validating and improving survey questionnaires in healthcare settings. ${ }^{33}$ The 
interviews will be supplemented by data on response rates and evaluation forms.

\section{Interviews sample}

Interviews will be conducted with patients from each ward included in the study $(\mathrm{n}=36)$. A representative sampling framework will be used to identify patients who reported a range of experiences of safety, were transported by different means and arrived or were admitted to different organisations. The inclusion criteria for interviews include:

- Able to give informed consent

- Aged 18 or above

- Able to speak English

\section{Procedure}

The interview questions will be structured into four themes: general health questions, general safety questions, care transfer questions and safety survey questions. The general health questions will explore healthcare experiences from the patients' perspective and the general safety questions will explore how safety is understood and their experiences of safety. The care transfer questions will allow for further discussion of the answers to the general safety questions, applied to care transfers specifically. Finally, the safety survey questions will explore the patients' perspectives on the survey, including the applicability of the questions to their experiences and why they chose their answers. The questions will also focus on barriers and enablers to providing useful feedback on their own safety within care transfers. The patient interview schedule will be refined iteratively throughout data collection.

\section{Analysis}

Once each interview is complete it will be transcribed verbatim, then coded and analysed systematically using qualitative analysis software. Thematic analysis will be used, which is flexible enough to allow for new themes to emerge. ${ }^{34}$ The patient interviews and healthcare professional interviews (discussed below) will be analysed independently of each other. Response rates will be compared between wards, allowing for analysis of nonresponse bias. Planning for this before the data collection commences is considered the best practice when conducting surveys within healthcare settings. ${ }^{35}$ Evaluation forms will be compared with responses on the safety survey using non-parametric statistical tests and descriptive statistics, and feedback will be used to iteratively modify the safety surveys on a monthly basis.

\section{Component 3: quality improvement using patient}

experiences of safety

Objective

Investigate the engagement and responsiveness of healthcare teams to the reporting tools and reports of safety, and the promotion of organisational learning.

\section{Method}

Semistructured interviews will be conducted with healthcare professionals who were involved in the transfer of patients or who received the patient feedback.

\section{Sample}

Four healthcare professionals from each of the included wards, including the ward manager, patient safety lead (where in post), discharge coordinator (where in post) and other ward-based staff involved in care transfer $(n=60)$, will be invited to participate in interviews or focus groups during the period of study. These participants will meet the following inclusion criteria:

- Work on one of the included wards during the period where safety surveys are distributed, where:

- They are responsible for managing the ward, or

- They have been involved in distributing the safety survey, or

- They have responsibility for discharging patients.

Healthcare professionals $(n=15)$ from other organisations (eg, ambulance services, nursing homes) involved in the journey or arrival/admission process of the care transfer, identified via the mapping process, will also be interviewed. The inclusion criterion for these participants is:

- Have responsibility for the management of patients or services relating to the transfer of the patient.

\section{Procedure}

The interview questions will be structured into three themes: general questions, general patient safety questions and questions about safety survey feedback. The general questions will enquire about professional background, current role and length of service in their current role and healthcare setting. The general patient safety questions will focus on understandings of patient safety, their perceptions of patient involvement in safety and the role of patients in reporting experiences of safety. The questions relating to the safety survey feedback will investigate how much contact they had with the feedback, how the feedback has been used in practice, including the relevance and appropriateness of information provided, and the barriers or enablers to using the feedback to learn about patients' perceptions of safety and improve services. This section will also contain the cognitive interview component, where participants will be asked how they interpret the domains of safety in the survey. The healthcare professional interview schedule will be refined iteratively throughout data collection.

\section{Analysis}

Once each interview is complete, it will be transcribed verbatim, then coded and analysed systematically using qualitative analysis software. Thematic analysis will be used, which is flexible enough to allow for new themes to emerge. ${ }^{34}$ 


\section{Mixed methods analysis}

Once data collection is complete across all objectives, mixed methods analysis will be conducted to provide a holistic meta-analysis. This analysis will consist of triangulating the findings in a convergence coding matrix, which displays findings from each component on the same page. ${ }^{36}$ The matrix will allow for an analysis of (dis)agreements, partial (dis)agreements or silences across the different components of the study, resulting in meta-themes.

This mixed methods analysis will provide a much deeper understanding of patient reporting of safety in care transfers than would have been possible if analysing the components in isolation. Case studies, underpinned by AI and combining the data together, will be developed to provide examples of best practice, relating to how patients are currently made to feel safe, or how the feedback has been used to improve safety and experiences of safety.

\section{Study management}

The study was designed with input from patient representatives from the Patient, Carer and Public Engagement network at the former North East Strategic Health Authority. The Patient, Carer and Public Engagement network was part of the Safer Care North East programme of work, where the safety survey was developed. One member of the network, along with two other patient representatives, will be part of an advisory group to provide direction and guidance throughout the study. Other members of the advisory group will include two healthcare professionals responsible for safety and quality at the included NHS Trusts, independent patient safety experts, the research team and senior healthcare professionals with expertise in healthcare policy.

\section{ETHICS AND DISSEMINATION}

Patient feedback received via the safety survey will be disseminated regularly to the healthcare teams and organisations involved in the study, in line with the quality improvement strategy, as stated in the study design section. Respondents will have the opportunity to request at the time of returning the survey a summary of the findings, which will be provided at the end of the study. The findings will also be published in peerreviewed journals and at academic and health services conferences.

\section{DISCUSSION}

This paper has presented the background, methodology and methods of a large scale, multisite research project investigating the feasibility for patients to provide feedback on their experiences of safety relating to their care transfer. As identified earlier, there is an increasing emphasis on the need for patients to be involved in their own safety, ${ }^{13} 1437$ highlighted by the recent events and subsequent enquiries at Mid-Staffordshire NHS Foundation Trust. ${ }^{16} 17$ This need can be addressed through patient reporting of safety concerns or incidents, ${ }^{30}$ so that organisations can learn from the patient perspective. This study will address that need in the context of care transfers.

Surveys are the most common method of soliciting patient reports of safety, ${ }^{13} 30$ although there has been little research into how the results can lead to organisational learning and quality and safety improvements. In settings other than patient safety, it has been identified that if used correctly, patient feedback can lead to improvements in the quality of care delivered, ${ }^{38} 39$ although this does not occur as often as it should. ${ }^{40}$

This study will build on this knowledge base by closing the gap between patients being involved in traditional incident reporting and the universal invitation for patients to provide feedback on their experiences of safety. This will also allow for the investigation of how patient feedback on safety can instigate quality improvements within multiple healthcare providers when patients' care crosses healthcare boundaries: a time when patients are at particularly high risk of experiencing safety incidents. ${ }^{89} 11$ Depending on the outcomes of the study, such as whether patient feedback on their experiences of safety can lead to improvements and how this feedback relates to other measures and indicators of quality and safety, there is potential for this feedback to become routinely collected data.

As with other studies on patient involvement in safety, ${ }^{41}$ there are a number of risks to the study which can be broadly categorised into patient engagement and healthcare professional engagement. While evidence suggests that patients can be involved in their safety when willing and able ${ }^{37}$ the majority of this research has been conducted within single healthcare settings such as a hospital, and transferability of findings to care transfers is unknown. The distribution of the reporting tool during discharge, alongside other discharge documentation, has the potential to result in information overload among patients, which may reduce response rates. This may be further complicated as older patients often do not understand discharge information. ${ }^{42}$ Proactive dialogue between the research team and healthcare professionals can help to mitigate this by encouraging the healthcare professionals to highlight the purpose of the survey to patients, thus encouraging dialogue around discharge information. The potential for reduced response rates will be further mitigated by distributing the survey universally, although this does not entirely remove the risk that data collected does not reflect the whole population being surveyed, but only those who are able and willing to respond. As with other patient involvement in safety research, ${ }^{43}$ the research team acknowledges that ultimate responsibility for patients' safety remains with the healthcare teams, and the safety feedback is meant to supplement rather than replace other measures and indicators of quality and safety. 
In a similar manner, the patient safety surveys are not a means to quality improvement alone, but instead are part of a larger process of organisational learning and continuous quality improvement, which incorporates feedback loops and the constant analysis of data. Consequently, and as the research team will not be imposing themselves on the healthcare professionals in the sense of active encouragement of team meetings or having a constant presence within their teams, it is possible that the feedback will be overlooked or ignored. This risk can be mitigated by including proactive dialogue between the research team, healthcare teams and the patient safety managers within the research sites outside of the feedback periods, discussing inside and outside the interviews any issues that arise. Understanding any barriers to engagement is also important to uptake, and this dialogue should help to improve the ecological validity of the safety surveys. $^{38}$

The authors intend to demonstrate how, or if, routinely collected data on patient experiences of safety is linked to other measures and indicators of quality and safety, much like patient incident reporting shares some similarities with healthcare professional incident reporting. ${ }^{19-21}$ The investigation of the processes involved in using and learning from this feedback will provide a greater understanding of how improvements can be made to quality and safety as a result of patient feedback, within the context of care transfers.

Acknowledgements The research team would like to thank The Health Foundation, an independent charity working to improve the quality of healthcare in the UK, for funding the study. We would also like to thank the National Health Service (NHS) Trusts involved in the study for their ongoing support, and members of the advisory group who have helped to guide the project. The research team also acknowledge the support of the National Institute for Health Research, through the Comprehensive Clinical Research Network.

Contributors JS, JW and PD were involved in the conception and design of the study and preparation of the manuscript. EH was involved in preparation of the manuscript. All the authors read and approved the final manuscript.

Competing interests None.

Ethics approval The study has been granted ethical approval by Yorkshire \& The Humber-Leeds West NHS Ethics Committee (13/YH/0372).

Provenance and peer review Not commissioned; peer reviewed for ethical and funding approval prior to submission.

Open Access This is an Open Access article distributed in accordance with the Creative Commons Attribution Non Commercial (CC BY-NC 4.0) license, which permits others to distribute, remix, adapt, build upon this work noncommercially, and license their derivative works on different terms, provided the original work is properly cited and the use is non-commercial. See: http:// creativecommons.org/licenses/by-nc/4.0/

\section{REFERENCES}

1. Department of Health. An organisation with a memory: report of an expert group on learning from adverse events in the NHS. London: Department of Health, 2000.

2. Kohn LT, Corrigan JM, Donaldson MS. To err is human: building a safer health system. Washington, DC: National Academies Press, 2000.
3. Donaldson L. An international language for patient safety: global progress in patient safety requires classification of key concepts. Int J Qual Health Care 2009;21:1.

4. Reason J. Human error: models and management. BMJ 2000;320:768-70

5. Waring JJ. Beyond blame: cultural barriers to medical incident reporting. Soc Sci Med 2005;60:1927-35.

6. Albolino S, Tartaglia R, Bellandi T, et al. Patient safety and incident reporting: survey of Italian healthcare workers. Qual Saf Health Care 2010;19(Suppl 3):i8-12.

7. Sari AB-A, Sheldon TA, Cracknell A, et al. Sensitivity of routine system for reporting patient safety incidents in an NHS hospital: retrospective patient case note review. BMJ 2007;334:79.

8. Forster AJ, Murff HJ, Peterson JF, et al. The incidence and severity of adverse events affecting patients after discharge from the hospital. Ann Intern Med 2003;138:161-7.

9. Forster AJ, Clark HD, Menard A, et al. Adverse events among medical patients after discharge from hospital. CMAJ 2004;170:345-9.

10. Kripalani S, LeFevre F, Phillips CO, et al. Deficits in communication and information transfer between hospital-based and primary care physicians: implications for patient safety and continuity of care. JAMA 2007;297:831-41.

11. Cook RI, Render M, Woods DD. Gaps in the continuity of care and progress on patient safety. BMJ 2000s;320:791-4.

12. Groene RO, Orrego C, Suñol R, et al. 'It's like two worlds apart': an analysis of vulnerable patient handover practices at discharge from hospital. BMJ Qual Saf 2012;21(Suppl 1):i67-75.

13. King A, Daniels J, Lim J, et al. Time to listen: a review of methods to solicit patient reports of adverse events. Qual Saf Health Care 2010;19:148-57.

14. Scott J, Dawson P, Jones D. Do older patients' perceptions of safety highlight barriers that could make their care safer during organisational care transfers? BMJ Qual Saf 2012;21:112-17.

15. Flink M, Hesselink G, Pijnenborg L, et al. The key actor: a qualitative study of patient participation in the handover process in Europe. BMJ Qual Saf 2012;21(Suppl 1):i89-96.

16. Francis R. The Mid Staffordshire NHS Foundation Trust Public Inquiry. Norwich: The Stationery Office, 2013.

17. National Advisory Group on the Safety of Patients in England. $A$ promise to learn-a commitment to act: improving the safety of patients in England. London: Williams Lea, 2013.

18. Hall J, Peat M, Birks Y, et al. Effectiveness of interventions designed to promote patient involvement to enhance safety: a systematic review. Qual Saf Health Care 2010;19:1-7.

19. Friedman SM, Provan D, Moore S, et al. Errors, near misses and adverse events in the emergency department: what can patients tell us? CJEM 2008; 10:421-7.

20. Weissman JS, Schneider EC, Weingart SN, et al. Comparing patient-reported hospital adverse events with medical record review: do patients know something that hospitals do not? Ann Intern Med 2008;149:100-8.

21. Zhu JY, Stuver SO, Epstein AM, et al. Can we rely on patients reports of adverse events? Med Care 2011;49:948-55.

22. Cooperrider DL, Srivastva S. Appreciative inquiry in organizational life. Res Organ Change Dev 1987;1:129-69.

23. Cooperrider DL, Whitney D, Stavros JM. Appreciative inquiry handbook: the first in a series of Al workbooks for leaders of change. Brunswick, OH: Crown Custom Publishing, Inc, 2005.

24. Reed J. Appreciative inquiry: research for change. London: Sage, 2007.

25. Shojania KG, Thomas EJ. Trends in adverse events over time: why are we not improving? BMJ Qual Saf 2013;22:273-7.

26. Hollnagel E. Thought paper: proactive approaches to safety management. London: The Health Foundation, 2012.

27. Trajkovski S, Schmied V, Vickers M, et al. Implementing the 4D cycle of appreciative inquiry in health care: a methodological review. $J$ Adv Nurs 2013;69:1224-34.

28. Tsilimingras D, Brummel-Smith $\mathrm{K}$, Brooks RG. Postdischarge adverse events in the elderly. J Patient Saf 2009;5:201-4.

29. Darzi A. High quality care for all: NHS next stage review (final report). London: Department of Health, 2008.

30. Masso Guijarro P, Aranaz Andres JM, Mira JJ, et al. Adverse events in hospitals: the patient's point of view. Qual Saf Health Care 2010;19:144-7.

31. McColl E, Jacoby A, Thomas L, et al. Design and use of questionnaires: a review of best practice applicable to surveys of health service staff and patients. Health Technol Assess 2001:5:1-256.

32. Schultze $U$, Avital M. Designing interviews to generate rich data for information systems research. Inf Organ 2011;21:1-16. 
33. Drennan J. Cognitive interviewing: verbal data in the design and pretesting of questionnaires. J Adv Nurs 2003;42:57-63.

34. Braun V, Clarke V. Using thematic analysis in psychology. Qual Res Psych 2006;3:77-101.

35. Halbesleben JRB, Whitman MV. Evaluating survey quality in health services research: a decision framework for assessing nonresponse bias. Health Serv Res 2013;48:913-30.

36. O'Cathain A, Murphy E, Nicholl J. Three techniques for integrating data in mixed methods studies. BMJ 2010:341:c4587.

37. Davis RE, Sevdalis N, Vincent CA. Patient involvement in patient safety: how willing are patients to participate? BMJ Qual Saf 2011:20:108-14.

38. Riiskjær E, Ammentorp J, Nielsen JF, et al. Patient surveys-a key to organizational change? Patient Educ Couns 2010;78:394-401.

39. Reeves $R$, West $E$, Barron D. Facilitated patient experience feedback can improve nursing care: a pilot study for a phase III cluster randomised controlled trial. BMC Health Serv Res 2013;13:259.

40. Wiig S, Storm M, Aase K, et al. Investigating the use of patient involvement and patient experience in quality improvement in Norway: rhetoric or reality? BMC Health Serv Res 2013;13: 206

41. Ward J, McEachan R, Lawton R, et al. Patient involvement in patient safety: protocol for developing an intervention using patient reports of organisational safety and patient incident reporting. BMC Health Serv Res 2011;11:130.

42. Hastings SN, Barrett A, Weinberger M, et al. Older patients' understanding of emergency department discharge information and its relationship with adverse outcomes. J Patient Saf 2011;7:19-25.

43. Davis RE, Jacklin $\mathrm{R}$, Sevdalis $\mathrm{N}$, et al. Patient involvement in patient safety: what factors influence patient participation and engagement? Health Expect 2007;10:259-67. 